\title{
Correlation between bulk stresses and interparticle contact forces in fine powders
}

\author{
M. A. S. Quintanilla, A. Castellanos, and J. M. Valverde \\ Departamento de Electronica y Electromagnetismo, Facultad de Fisica, Avenida Reina Mercedes s/n, 41012 Sevilla, Spain
}

(Received 9 February 2001; published 13 August 2001)

\begin{abstract}
We present measurements of the tensile strength as a function of the consolidation stress for a set of fine cohesive powders (xerographic toners) of $12.7 \mu \mathrm{m}$ particle size and with a range of concentration of submicron fumed silica as flow control additive. This additive is well known for its ability to control interparticle adhesion force. Parallel measurements using an atomic force microscope have been carried out on the adhesion force between two individual grains as a function of a controlled previous load force. The effect of the additive on the tensile strength and adhesion force is analyzed. We have found a good correlation between bulk stresses and adhesion forces between individual particles. This correlation is compatible with the existence of a subnetwork of force chains.
\end{abstract}

DOI: 10.1103/PhysRevE.64.031301

PACS number(s): 45.70.-n, 81.05.Rm, 83.80.Fg

\section{INTRODUCTION}

A large part of industries that deal with fine powders (particle size $\leqslant 30 \mu \mathrm{m}[1])$ are in some way affected by flowability problems. The flow of fine powders is dominated by the interparticle contact forces $[1,2]$ that are very sensitive to the previous history of the material $[3,4]$. By means of a powder bed technique [5] we measured the tensile strength and the solid volume fraction of a set of fine powders as a function of the previous consolidation stress. Those measurements served us to evaluate the effect of flow control additives [4] and particle size [6,7] on the sample flowability. Additionally, we estimated from these macroscopic variables the interparticle contact forces under the assumption of a continuous and isotropic distribution of stresses inside the material. On the other hand, several authors $[8,9]$ have reported on direct measurements of the adhesion force between individual grains. Nevertheless, up to our knowledge, the measured microscopic forces have not been related to the external bulk stresses on the granular assembly. In the present work we measure the adhesion force between two loaded individual grains by means of an atomic force microscope (AFM) and compare the results with the interparticle forces estimated from the measured tensile strength and consolidation stress of the bulk material. To this end some kind of relationship must be adopted to estimate contact forces form bulk stresses. Indeed, there is an extensive work in the literature where the microscale variables (contact forces) and the macroscale variables (stresses) are linked. On one hand, homogenization techniques have been used to derive the stress tensor of the material from a model for the interparticle contact and a contact distribution function [10-13]. The resulting "averaging" relationships are built under the approach of continuum mechanics. On the other hand, our interest will focus on the derivation of the contact forces from the bulk stresses, which has been addressed in several theoretical works $[11,14-16]$. Following the nomenclature of Emeriault and Chang [11] we will refer to this problem as the "tracking problem."

\section{A. The tracking problem}

The problem of "tracking," i.e., the derivation of the interparticle contact forces from the bulk stresses, dates back to the early work of Rumpf [14], who obtained a relationship between the isotropic, normal stress and the interparticle contact force from simple geometrical arguments. For a system of hard monodisperse spherical particles with a random isotropic packing, a relation

$$
F=\frac{\pi d_{p}^{2}}{\phi k} \sigma
$$

was established between the contact force $F$ and the hydrostatic stress $\sigma$. Here $d_{p}$ is the particle diameter, $k$ is the coordination number, which is defined as the average number of contacts per particle, and $\phi$ is the solid volume fraction. Some decades later, Helle et al. [15] arrived at the same relationship by using the principle of virtual work. More recently [11] the effect of an anisotropic distribution of contacts has been considered. From the theorem of virtual works, Emeriault and Chang [11] showed that the stress tensor $\sigma_{i j}$ and the interparticle contact force $F_{i}$ can be related by the equation

$$
F_{i}=\sigma_{i j} n_{k} A_{k j},
$$

where $n_{i}$ is a unit vector parallel to the contact orientation, and $A_{i j}$ is the inverse of the fabric tensor. The fabric tensor $F_{i j}$ is defined as [11]

$$
F_{i j}=\frac{d_{p}}{V} \sum_{c} n_{i}^{c} n_{j}^{c} .
$$

The summation extends over all contacts in the volume $V$ and $n_{i}^{c}$ are the unit vectors normal to the surface of the particles at the contact points. In their derivation Emeriault and Chang [11] assumed that all contacts with the same orientation carry the same contact force, i.e., there is not a contact force distribution for the population of contacts in a given orientation. Under this hypothesis, if the contact angular distribution and the coordination number are known, the fabric tensor can be calculated and Eq. (2) can be used to derive contact forces from stresses. In the particular case of an isotropic contact angular distribution and a uniaxial stress tensor, Eq. (1) is recovered. In the Appendix we have considered the effect of an anisotropic contact angular distribution 
resulting from a uniaxial compression of the powder. We arrive then at (see the Appendix)

$$
F=\frac{\pi d_{p}^{2}}{\phi k} \sigma\left(1+\frac{2}{\sqrt{5}} \zeta\right)^{-1},
$$

which differs from Eq. (1) only in a multiplicative factor. In a two-dimensional (2D) system $\zeta \sim 0.1$ [17], which means only a $10 \%$ decrease in the contact force. Hopefully this correction will not be much higher for a 3D system.

The effect of polydispersity has also been addressed in the literature. Using the principle of virtual work, Tsoungui et al. [16] deduced the mean normal force $F$ on a contact as a function of the external stress $\sigma$ for a polydiperse 3D system

$$
F=\frac{\pi d_{p}^{2}}{\phi k} \sigma \beta,
$$

where $\beta=1$ for a monodisperse packing, thus recovering Eq. (1). The parameter $\beta \geqslant 1$ was shown to be a function of the variation of the density of the packing with the mean particle volume [16]. For a bimodal distribution in a 3D system, $\beta$ presents a maximum value of 1.5 for a small particle volume fraction close to 0.2 , but, in general, displays a weak dependence on the particle size ratio. Our experimental powders are rather monodisperse and we expect that this correction will be minor. Thus, the effects of anisotropy and polydispersity are the inclusion of a multiplicative factor close to unity in Eq. (1). In what respect the irregularities of particles surface we have not found any realistic approach that deals with this effect and we will ignore it.

In the derivation of the above equations it is assumed that the medium can be described as a continuum. However numerous experiments have demonstrated that particles in granular materials are unequally involved in the transmission of the stresses [18-23]. Particles subjected to load forces higher than the mean form force chains that sustain most of the deviatoric stresses inside the material whereas the rest of the particles are subjected practically to a homogeneous pressure [17]. Large fluctuations in stresses deviate strongly from the mean values in a scale of tens to hundreds of grains $[19,20,23,24]$, perhaps large enough to invalidate a continuum description of the granular material. Force chains have been revealed in a number of papers reporting measurements of contact forces along the confining walls of the assembly. Liu et al. [18] showed that the probability distribution function $P(f) \quad(f=F / \bar{F})$ decays exponentially for normal forces $F$ larger than the mean $\bar{F}$ while it becomes more or less constant for $f<1$. Similar results have been obtained by Mueth et al. [21] and by Lovoll et al. [22]. This characteristic exponential decay for large forces has been also associated with the stresses transmitted in the slow shearing for both 3D and 2D Couette flows [23]. The exponential tail of the distribution can be understood on the basis of a statistical model ( $q$ model) [25], where forces on a particle are provided from neighbors in a random way. In this model friction is neglected and the disorder of the packing is considered as the dominant physical mechanism leading to force chains. It has also been found by contact dynamics simulations [24] that the normalized distribution of normal forces collapses on the same distribution independent of particle size and size dispersity. In a recent work [26] simulations on the evolution of the contact normal force distribution were performed on a system of slightly cohesive particles. The low cohesion introduced between particles did not change the main features of the force distribution compared with noncohesive systems. Blair et al. [27] have just presented a systematic experimental study on the distribution of normal forces at the bottom of static packings of spheres. They have shown that the force distribution remained essentially unaffected when the bead pack was varied from amorphous to crystalline and when the interparticle coefficient of friction was changed. We believe that the possible existence of stress networks in our fine powders at a macroscopic scale may have a non-negligible influence on the estimation of the interparticle adhesion force from the measured tensile strength. This issue will be discussed later.

Equation (1) involves the coordination number $k$ that has been related to the solid volume fraction $\phi$ by means of numerical simulations of ballistic deposition in which various degrees of restructuring are allowed [28]. For our powders $k$ is in the range $2<k<4$ and $\phi$ lies between 0.2 and 0.4. In this range, numerical results can be approximated by the equation

$$
k \simeq 1.25(1-\phi)^{3 / 2} .
$$

From Eqs. (1) and (6) the average load force $P$ on the interparticle contacts and the average adhesion force $F_{t}$ of the interparticle contacts will be roughly estimated from the measured bulk consolidation stress $\sigma_{c}$ and tensile strength $\sigma_{t}$, respectively,

$$
\begin{aligned}
& P \sim \frac{\pi d_{p}^{2}}{1.25 \phi}(1-\phi)^{-3 / 2} \sigma_{c}, \\
& F_{t} \sim \frac{\pi d_{p}^{2}}{1.25 \phi}(1-\phi)^{-3 / 2} \sigma_{t} .
\end{aligned}
$$

\section{MATERIALS}

We have run the experiments with two different xerographic toners that are identical in particle resin type (styrene butadiene), pigment concentration, average particle volume diameter $\left(d_{p}=12.7 \mu \mathrm{m}\right)$, and particle density $\left(\rho_{p}\right.$ $=1.065 \mathrm{~g} / \mathrm{cm}^{3}$ ). Toner particles are irregular (see Fig. 1) since they are formed by a grinding process and they are rather monodisperse. Both powders differ only in the quantity of flow controlling additive (submicron fumed silica particles). This additive (Aerosil R812) consists of agglomerates of nanoparticles of fumed silica. From scanning electron microscopy (SEM) pictures of the toner particles (see Fig. 2), we have observed that the individual additive particle has a diameter of $7 \mathrm{~nm}$ and that the additive particles are distributed in agglomerates that are randomly placed on the powder surface. These agglomerates have estimated diameters ranging from 40 to $60 \mathrm{~nm}$. Additives were added in the amount 


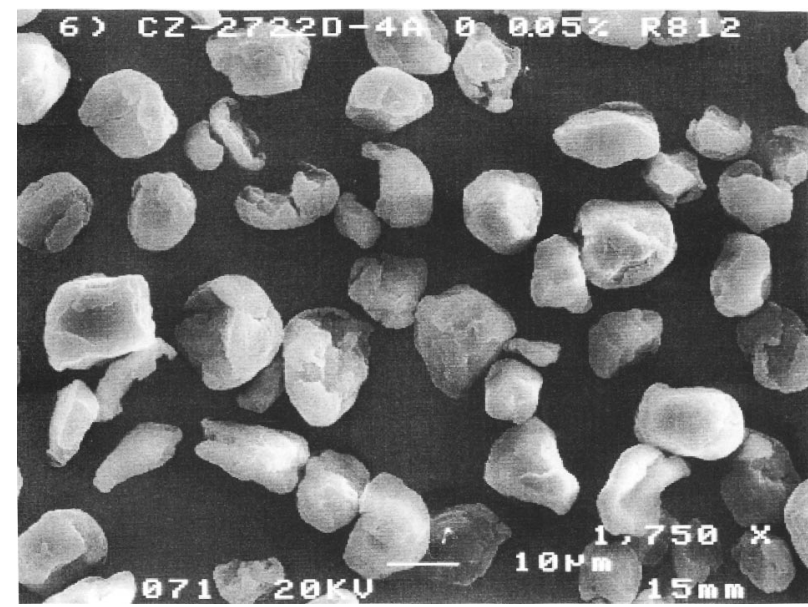

FIG. 1. SEM micrograph of toner particles. The bar size is $10 \mu \mathrm{m}$.

necessary to produce a theoretical surface area coverage of $80 \%$ (0.4 wt. \%, toner RT-5117-2) and $10 \%$ (0.05 wt. \%, toner RT-5114-3) of the total surface of the particles.

The effect of Aerosil on the powder tensile strength and packing fraction is described in our previous paper [4]. The addition of these nanoparticles results in a reduction in the powder tensile strength because the additives are made of a hard material and therefore they increase the hardness of the contacts; they also reduce the powder tensile strength by reducing the size of the contacts. A higher additive level increases the ability of the particles to rearrange themselves in more packed structures because of the reduction in powder cohesivity. Despite the reduction of cohesivity caused by additives, interparticle attractive forces can exceed in several orders of magnitude the particle weight [1]. As a consequence cohesive effects are dominant in the bulk behavior [2]. Adhesive forces oppose very efficiently gravity and particles settle in very open structures $(\phi \sim(0.2,0.4))[28]$. The strong cohesivity of fine powders enhances packing inhomogeneities and this in turn could favor the development of force chains at a large scale compared to particle size.

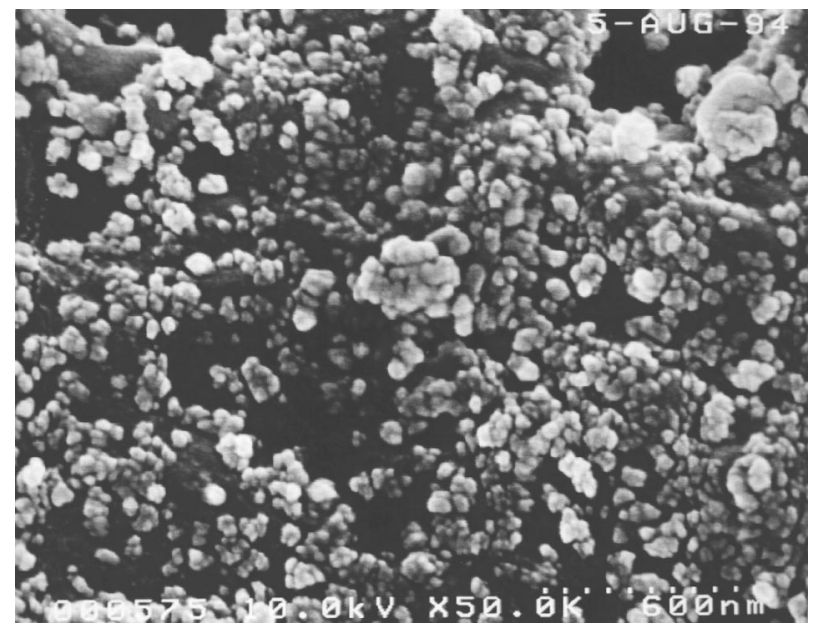

FIG. 2. SEM micrograph of the surface of a powder particle showing the distribution of the particles of flow control additive over the particle surface.

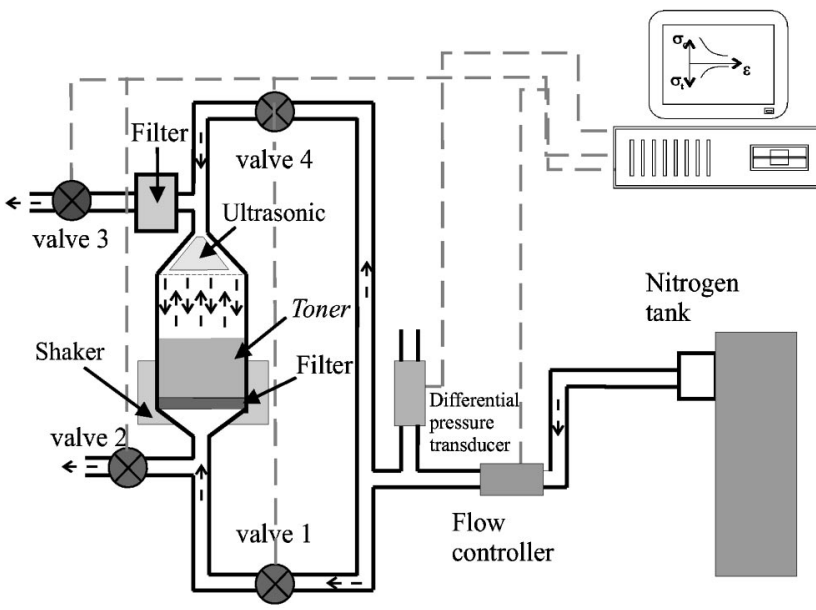

FIG. 3. Experimental setup for the measurement of the tensile strength as a function of the consolidation stress.

\section{BULK STRESSES AND ESTIMATED CONTACT FORCES}

A schematic view of the experimental system for measuring the tensile strength $\sigma_{t}$ as a function of the consolidation stress $\sigma_{c}$ is shown in Fig. 3. The sample of powder is held in a cylindrical container that rests on a porous filter of sintered metal particles ( $5 \mu \mathrm{m}$ pore size). The container is placed over a shaker to vibrate the powder when fluidized to avoid plugging. A set of electrical valves and a flow controller control the flow of dry nitrogen through the system while a differential manometer monitors the gas pressure drop across the powder bed. A computer controls all the setup so the experiment and data acquisition is fully automatized. In all our measurements we initialize the sample by fluidizing the powder in the bubbling regime. After initialization, the sample is compressed against the filter with a downward flow of nitrogen or allowed to collapse in an upward flux of gas depending on the desired value for the consolidation stress at the bottom (larger or smaller than the consolidation stress of the sample due to its own weight per unit area). The value of the consolidation stress at the sample bottom is the powder weight per unit area plus or minus (depending on the use of the gas flow to compress or decompress) the gas pressure drop across the powder bed. An ultrasonic sensor measures the height of the powder bed to calculate its solid volume fraction. The next step is to break the sample with an upward flow of gas. To obtain the tensile strength the gas flow is increased slowly from zero. The bed at first remains undisturbed and the pressure drop across the powder is proportional to the gas flow according to Carman's law [29] (see Fig. 4). As the gas velocity is increased further, a point is reached at which the pressure drop becomes equal to the weight per unit area of the sample. When the gas velocity is further increased the pressure drop continues to increase until interparticle cohesive forces are overcome and a falloff in the pressure drop is observed (see Fig. 4). This drop coincides with a visible fracture at the bottom of the bed. We have observed that after the fracture a thin layer of powder always remain in the filter. This indicates that fracture occurs in the powder and not at the powder-filter interface. The excess of 


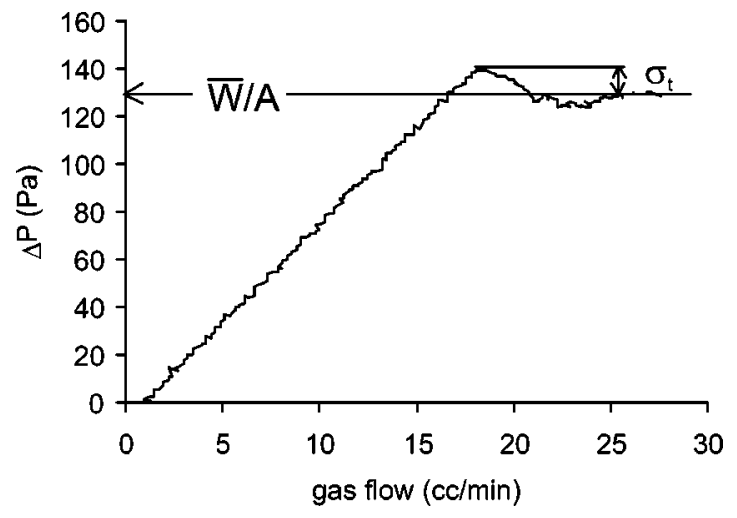

FIG. 4. Typical curve for the pressure drop across the powder bed vs gas flow (xerographic toner with particle size $12.7 \mu \mathrm{m}$, $0.4 \%$ by weight of flow additive). The overshoot of the pressure drop beyond the weight per unit area of the bed $(\simeq 130 \mathrm{~Pa})$ is taken as the tensile strength of the powder $\left(\sigma_{t} \simeq 10 \mathrm{~Pa}\right)$.

the pressure drop over the weight per unit area of the bed is taken as the tensile strength of the sample. A more detailed description of the experimental setup and procedure can be found elsewhere [5].

In Fig. 5 we present results of the tensile strength versus the previous consolidation stress for both powders investigated. As we have already shown [4] the tensile strength increases with the applied consolidation stress. The increase in the tensile strength is faster for the powder with 0.05 wt. $\%$ of additive than for the powder with 0.4 wt. $\%$ of additive due to the different nature of the contacts in both powders. For a given consolidation, the powder with 0.05 wt. $\%$ additive is more cohesive than the powder with 0.4 wt. \% additive because in the latter the majority of contacts are formed between particles of additive (silica-silica); whereas in the former the contacts polymer-polymer predominate. We have checked that the tensile stress needed to break the powder does not depend on the rate of increase of the gas flow. The same result is obtained when we break the powder by an instantaneous increase of gas flow or when the gas flow is increased quasistatically (see Fig. 6). Stresses are measured

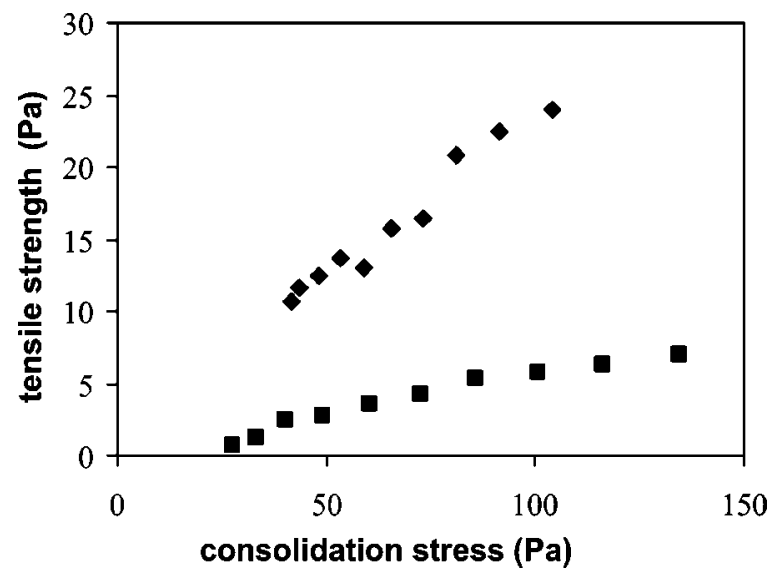

FIG. 5. Tensile strength $\sigma_{t}$ vs consolidation stress $\sigma_{c}$. Powder with 0.4 wt. $\%$ of additive (boxes). Powder with 0.05 wt. $\%$ of additive (diamonds).

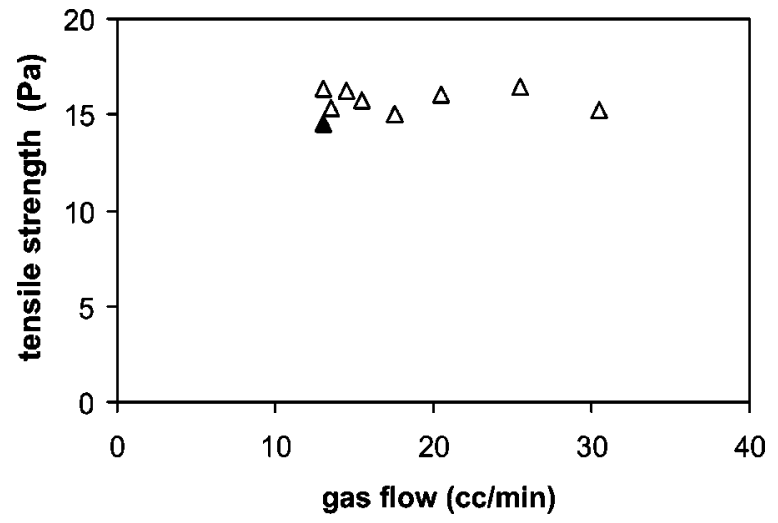

FIG. 6. Tensile strength $\sigma_{t}$ measured by increasing quasistatically the gas flow (solid triangle) and by imposing an instantaneous value of the gas flow (void triangles).

quite precisely (the estimated dispersion is around $2 \mathrm{~Pa}$ ). Furthermore, if the powder is compressed by a gravity directed gas flow, the consolidation stress at the bottom, given by the gas pressure drop plus the weight per unit area, increases monotonically. There are no strong fluctuations that may arise from any influence of stress chaining. Indeed, the powder area that is subjected to stress is $\simeq 15 \mathrm{~cm}^{2}$, which includes a number of $\sim 10^{8}$ particles. For such macroscopic system, the Mohr-Coulomb continuum theory would predict reasonably well the powder bulk properties. Otherwise, we found elsewhere [2] large fluctuations in the width of a powder avalanche when a layer of powder was quasistatically tilted and reached the maximum stable angle. The typical width of an avalanche was of the order of $1 \mathrm{~mm}$ [2], implying an order of $10^{2}$ particles, where the effect of stresses discontinuities can be significative. Thus, although force chains do not affect the measurement of the bulk tensile and consolidation stresses, they can be an important factor to take into account for the estimation of contact forces from the macroscopic stresses. Despite this and due to the impossibility of quantifying the influence of chaining, Eqs. (7) and (8) will be employed as a first approximation. The results for the estimated adhesion and load forces on individual contacts are plotted in Fig. 7. As it is well known from the adhesion theory of elastic solids [30] if the deformation of the particles were elastic, the adhesion force threshold between individual particles would be independent of the previous load force. Thus the data suggests that the interparticle contacts undergo either plastic or elastoplastic deformation but not elastic deformation. It must be noted that in the experiment the granular assembly is subjected to an edometric test. In order to use Eq. (1) we approximate the real stress state of the material to an uniaxial tension or compression along the vertical direction.

\section{MEASUREMENT OF ADHESION FORCES}

The sketch of AFM used for measuring the adhesion force between two individual grains is shown in Fig. 8. The probe particle is attached at the end of a " $\mathrm{V}$ " shaped tipless cantilever. To attach the particle to the cantilever, the particles are spread over a flat substrate. With the aid of an optical micro- 


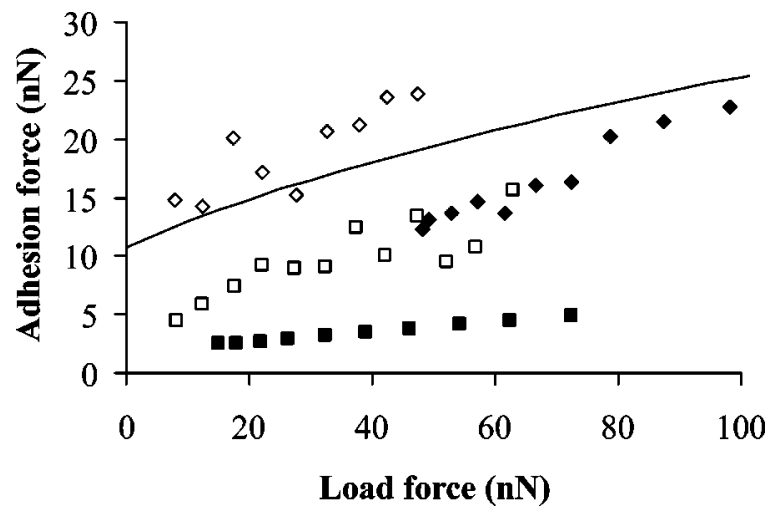

FIG. 7. Average (median value) adhesion vs load force measured from the AFM (void symbols) and estimated from bulk stresses (solid symbols). Powder with 0.4 wt. $\%$ of additive (boxes). Powder with 0.05 wt. $\%$ of additive (diamonds). The continuous line represents the theoretical prediction by the MP model [Eq. (13)] for a contact between polymer particles.

scope we choose an isolated particle and the cantilever is brought slowly close to it. At certain small separation, the particle jumps towards the cantilever and remains attached to it due to the adhesive forces between the powder particle and the cantilever (an indication that these forces may have an electrostatic origin is that particles show a tendency to jump towards the tip of the "V"). Although these forces are strong enough to keep the particle attached to the cantilever, they cannot prevent the probe particle from being removed if we make an indentation experiment with a substrate particle. In order to keep the probe particle permanently attached to the cantilever, the piece holding the cantilever with the particle on it is heated to sinter the contacts between particle and cantilever controlling the temperature to avoid melting the particle or changing its shape. The substrate particles are spread over a piece of transparency film and fixed to it permanently by heating them in the same way. The probe particle is brought close to an isolated substrate particle on the film under computer control. The computer detects the movement on the cantilever and stops the approach when the cantilever starts to bend. We place the probe particle very close to the substrate particle, but avoiding contact between them. The substrate particle is then retracted slightly, moved vertically by means of the AFM piezoelectric tube. At the beginning of a loading-unloading curve both particles are apart (point $A$ of Fig. 8). The substrate particle is moved upwards till it makes contact with the probe particle (point
$B$ ) and is pushed until a certain cantilever deflection is achieved (point $C$ ). Upward deflection of the cantilever means positive load force. After that, the substrate particle is slowly withdrawn. Both particles remain in contact causing the cantilever to bend downwards (negative load force or tensile force), until the substrate particle is detached (point $D)$. The largest downward deflection achieved by the cantilever gives the adhesion force. The piezoelectric extension and the current given by the split photodiode are recorded. The latter is transformed in nanonewtons assuming that both particles remain in contact while the piezoelectric is retracting until the "substrate" particle is detached. Neglecting particle deformation in the indentation process we obtain a proportionality constant that relates piezoelectric displacement and cantilever displacement, and from it and the spring constant of the cantilever the force exerted between the particle and the substrate is derived.

The number of data points collected for each powder is above 1000 in order to have confident statistics. The data forms a cluster with a certain dispersion that will be discussed later. These points have been ordered by increasing value of the load force and distributed in groups. Each point in Fig. 7 represents the average value of the adhesion for each group versus the load force. As can be seen from Fig. 7 the average measured adhesion force is about twice the estimated adhesion force in the whole range of load forces for both powders.

\section{THEORETICAL PREDICTION OF INTERPARTICLES FORCES}

If we assume that the deformation of the additive particles is negligible for small loads, we can use the Hamaker formula [31] for the van der Waals force of attraction between two spheres:

$$
F=\frac{A}{6 z^{2}}\left(\frac{1}{R_{1}}+\frac{1}{R_{2}}\right)^{-1}
$$

where $A$ is the Hamaker constant, $z$ is the minimum intermolecular distance ( $z \simeq 4 \AA$ [32]) and $R_{1}$ and $R_{2}$ are the radii of the two spheres. Most powder particles have a rather rough surface with many asperities of typical radius often not larger than $0.1 \mu \mathrm{m}[33,34]$. Thus the effective contact area is given by the asperities at contact and the interparticle attractive force can be approximately obtained [35] inserting the typical radius of the asperities $R_{a}$ in Eq. (9). In the case
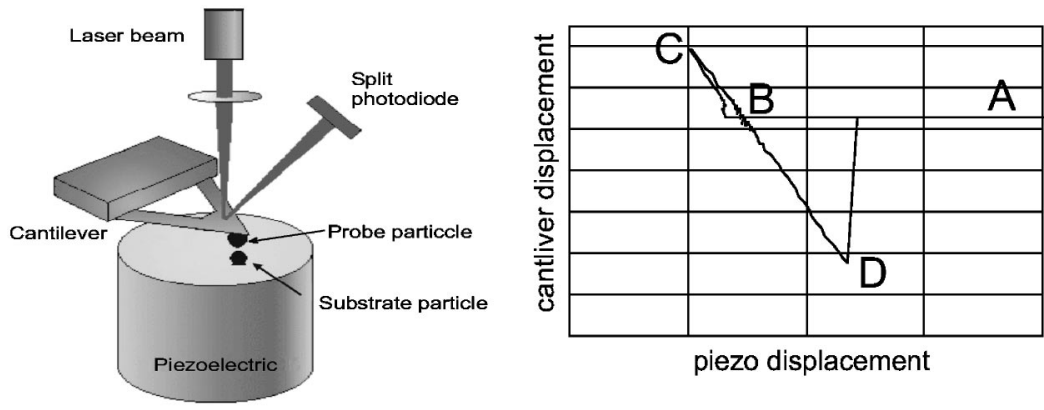

FIG. 8. Experimental setup for the measurement of interparticle forces with an atomic force microscope and a typical load-unload curve. 
of 0.4 wt. \% of additive concentration, the surface coverage of additives is around $80 \%$ and therefore we would expect that a large part of contacts is between additive aggregates. The size of the additive aggregates is estimated around 50 nm (Fig. 2). Assuming that we can take the van der Waals force of attraction as the adhesion force of the spheres in contact, Eq. (9) yields $F \simeq 2 \mathrm{nN}$ for two additive aggregates ( $A \simeq 1.5 \times 10^{-19} \mathrm{~J}$ for silica [36]), which agrees with the extrapolation of the average adhesion force obtained from the AFM measurements to zero load (Fig. 7). On the other hand, in the case of $0.05 \mathrm{wt} \%$ of additive concentration, the surface recovery of additives is only about $10 \%$ and therefore we should expect that the majority of contacts occur between polymer surfaces for which a typical asperity radius is $0.1 \mu \mathrm{m}$. Thus the predicted adhesion force is $F \simeq 3.4 \mathrm{nN}$ $\left(A \simeq 0.65 \times 10^{-19} \mathrm{~J}\right.$ for polymer [36]), which is somewhat below the extrapolation to zero load of the average adhesion force obtained from the AFM measurements (Fig. 7). This apparent disagreement can be explained by the effect of surface deformation induced by attractive forces that raises the adhesion force. It is known that strong attractive forces may originate by themselves a plastic deformation of the surfaces at contact [37-39]. This effect is more likely to appear between polymer contacts since polymer is softer than silica. By means of SEM Rimai et al. [37] were able to observe directly the adhesion-induced deformation of unloaded polystyrene smooth spheres on a rigid flat substrate. They found that the contact radius $a$ increased with the particle radius $r$ as $a \propto r^{0.42 \pm 0.13}$, in agreement with the Maugis-Pollock (MP) model [39], which assumes a plastic response. On the other hand, the results were inconsistent with the Johnson, Kendall, and Roberts model [40], which assumes that the particle responds elastically and predicts $a \propto r^{2 / 3}$.

According to the MP model [39], the relation

$$
P+2 \pi w r=\pi a^{2} H
$$

holds for a fully plastic contact, where $H$ is the hardness of the material, $w$ is the work of adhesion, $a$ is the radius of the area of contact, $P$ is the externally applied load force on the contact and $r$ is the local radius of curvature of the surfaces at contact. The hardness $H$ of the material is related to the yield strength $Y$ by $H=3 Y$. The work of adhesion is the energy required to separate a unit area of the surfaces in contact. For two surfaces of the same material in contact, the work of adhesion is given by $w=2 \gamma, \gamma$ being the surface energy of the material. The second term of the left hand side of Eq. (10) takes into account the adhesive surface forces acting on the area of contact. To further investigate if the attractive forces are sufficiently large when two toner particles are in touch so as to exceed the elastic limit we can follow the arguments of Krupp [32] and Rimai et al. [37]. Krupp [32] demonstrated that for two flat surfaces in contact separated by a distance $z_{0}$ the pressure $p_{m}$ due to attractive forces is given by

$$
p_{m}=\frac{2 w}{z_{0}}
$$

Rimai et al. [37] estimated that Eq. (11) yields a good approximation for the average pressure in the case of two spheres in contact. Upon substituting the value of $z_{0}=4 \AA$ and $w=0.07 \mathrm{~J} / \mathrm{m}^{2}$ (a typical value for polymer surface energy is $\gamma \simeq 0.033 \mathrm{~J} / \mathrm{m}^{2}$ [38]) in Eq. 11, it is found that $p_{m}$ is approximately $0.3 \mathrm{GPa}$, which is in excess of the typical yield strength of the polymer ( $Y \simeq 0.1 \mathrm{GPa}$ [41]). Thus, it is likely that the toner particles with just $0.05 \%$ of silica additive deform plastically.

When the contact is plastic, with partial elastic recovery of the material upon unloading, rupture of the contact is similar to the rupture of an elastic contact of two spheres with radius $r_{f}$ greater than the initial radius $r$ (thus the effect of plastic deformation is to increase the local radius of curvature of the surfaces prior to separation) [39]:

$$
r_{f}=\frac{a^{3} K}{\pi a^{2} p_{m}}=\frac{a K}{\pi p_{m}} ; \quad \frac{1}{K^{2}}=\frac{1-\nu^{2}}{E^{2}}
$$

where $\nu$ is the Poisson ratio (for polymer $\nu \simeq 1 / 3$ ) and $E$ is the Young modulus of the material. In a plastic contact, $p_{m}$ equals the hardness $H$. The adhesion force $F_{t}$ can be taken as the force of adhesion of a sphere of radius $r_{f}$ in an elastic contact [39]. Using Eq. (10) the adhesion and load forces can be related

$$
F_{t}=\frac{3}{2} \pi w r_{f} \Rightarrow F_{t}=\frac{3 \pi w K}{2(\pi H)^{3 / 2}} \sqrt{P+2 \pi w r}
$$

where $r=R / 2$ for a contact between two spheres of radius $R$. From Eq. (13) we can estimate the adhesion force at zero load. Taking $w \simeq 0.07 \mathrm{~J} / \mathrm{m}^{2}$ [38], $E \simeq 6 \mathrm{GPa}$ and $H$ $\simeq 0.3 \mathrm{GPa}[41]$ as typical values for polymer, and an asperity radius of $0.1 \mu \mathrm{m}$, we obtain $F_{t}(P=0) \simeq 11 \mathrm{nN}$, which is about the extrapolation to zero load of the data obtained from the AFM measurements in the case of $0.05 \%$ of additive concentration. In Fig. 7 the predicted curve of the MP model (Eq. 13) is plotted together with the experimental results in the range of load forces investigated. As can be observed there is a reasonable agreement between experimental data and theoretical results.

For the toner particles with $0.4 \mathrm{wt} . \%$ of silica $(80 \%$ of particle surface coverage) the application of theory is not straightforward. In this case the majority of contacts occur between silica aggregates that cover a large part of the particle surface. The response of these aggregates to a load force is complicated. Indeed the dynamics of the aggregated silica particles in contact should be different from the dynamics of two individual silica particles. Furthermore, the silica aggregates are resting on the polymer particle that is softer than silica. For these reasons it is difficult to estimate if the adhesion induced deformation is elastic or plastic and therefore to have a quantitative prediction of the evolution of the adhesion force with the load force. Our measurements indicate that the rate of increase of the adhesion force $F_{t}$ with the load force $P$ decreases when the kind of contacts changes from polymer dominated ( $0.05 \mathrm{wt} \%$ of silica additive) to 
silica dominated ( 0.4 wt. \% of silica additive). Obviously this is the reason of the widely spread use of these flow additives in powder industry.

\section{DISCUSSION}

A comparison of the estimated values of the adhesion force from bulk stresses and the measured from the AFM (Fig. 7) shows that the average adhesion force is correlated to the estimated contact forces from macroscopic measurements. In both cases the adhesion increases with load force and the increase is faster for the powder with $0.05 \mathrm{wt} . \%$ additive than for the powder with $0.4 \mathrm{wt}$. \%. Furthermore the rate of increase of the average adhesion force (AFM data) is similar to the rate of increase of the estimated adhesion force. Nevertheless, although both sets of data are in the same order of magnitude, we obtain, independently of the load force, that the estimated force from the bulk is about one-half of the average value measured from the AFM. To look more precisely for this correlation between the values of adhesion forces obtained from both methods we have grouped the measured values of the adhesion from the AFM in ranges of load force. For each measurement of the adhesion from the AFM we have calculated a reduced adhesion $f$ as the ratio of the adhesion force measured to the value of the adhesion estimated from the macroscopic measurements for the same load force. The cumulative frequency distributions of the reduced adhesion in each range of load forces are shown in Fig. 9. As can be observed the cumulative frequency distribution is independent of the range of load forces. This means that the distribution function of the values of the adhesion measured from the AFM scales with the values of the adhesion estimated from the bulk stresses. These ratios are disposed around a central value that does not depend on the load force and is the same for the two powders investigated. In Fig. 10 we have plotted the probability distribution function $P(f)$ of finding a value of the reduced adhesion $f$. It is clear from this plot that both powders, in spite of their different cohesivity, follow the same distribution within the experimental scatter. This distribution has a peak around $f=2(\sim$ average value $)$ and is not symmetrical. Remarkably, it shows an exponential decay for forces above the average value. As mentioned in the Introduction the effects of anisotropy and polydispersity would introduce a factor close to one in the estimated forces and therefore do not play a significant role. On the contrary, the large fluctuations on forces on a macroscopic scale can be an important correction to the estimation of contact forces from the macroscopic stresses. The influence of a strong cohesivity on the distribution of forces remains to be investigated but we assume that the basic characteristics concerning chaining in noncohesive or slight cohesive granular materials are retained. Thus, if the application of a consolidation stress to the granular system leads to stress chaining, only a fraction of contacts carries in effect most of the applied external load while the rest of contacts do not feel the external load. If the measured bulk stress were distributed only over the contacts that do belong to the subnetwork of large forces, the estimated contact force $F^{\prime}$ would be larger than the estimated $F$
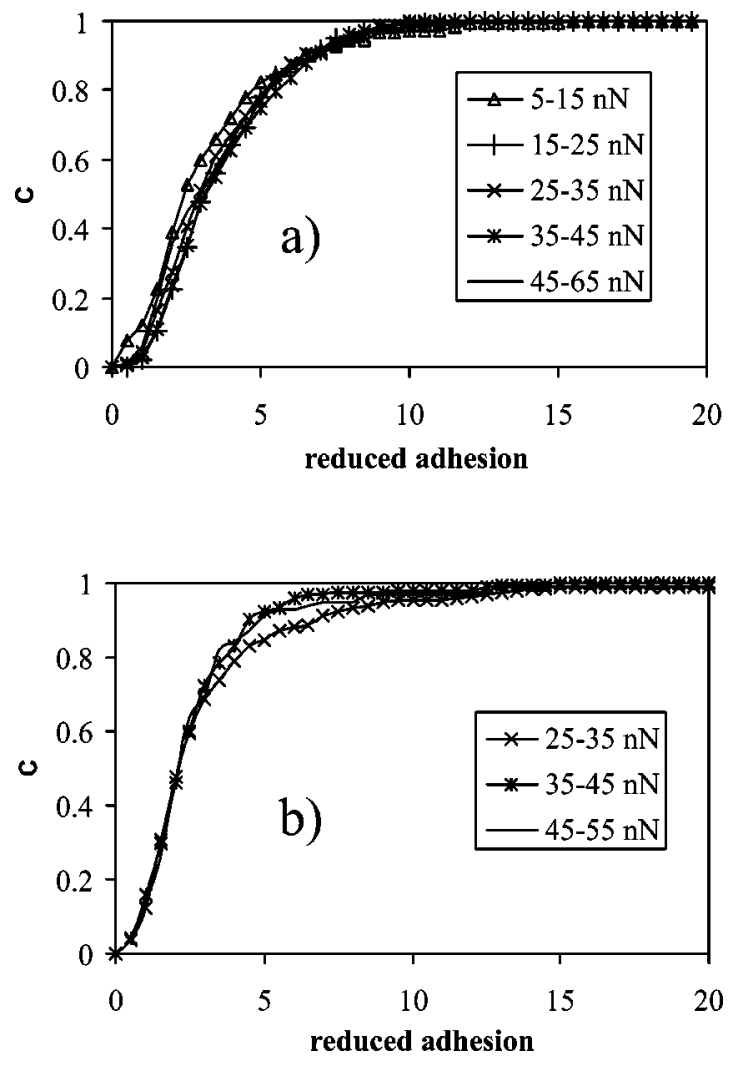

FIG. 9. Cumulative frequency distribution $C$ of the reduced adhesion for different ranges of load force (indicated in the inset). (a) powder with $0.4 \%$ of additive, (b) powder with 0.05 wt. $\%$ of additive.

from Eq. (1). In the simplest approach the average contact force $F^{\prime}$ estimated would scale as $F^{\prime} \sim F / \eta$, being $\eta<1$ the fraction of contacts that in effect carry the total external stress. If we assume that $\eta$ is constant and as long as the relationship between the adhesion force $F_{t}$ and the load force

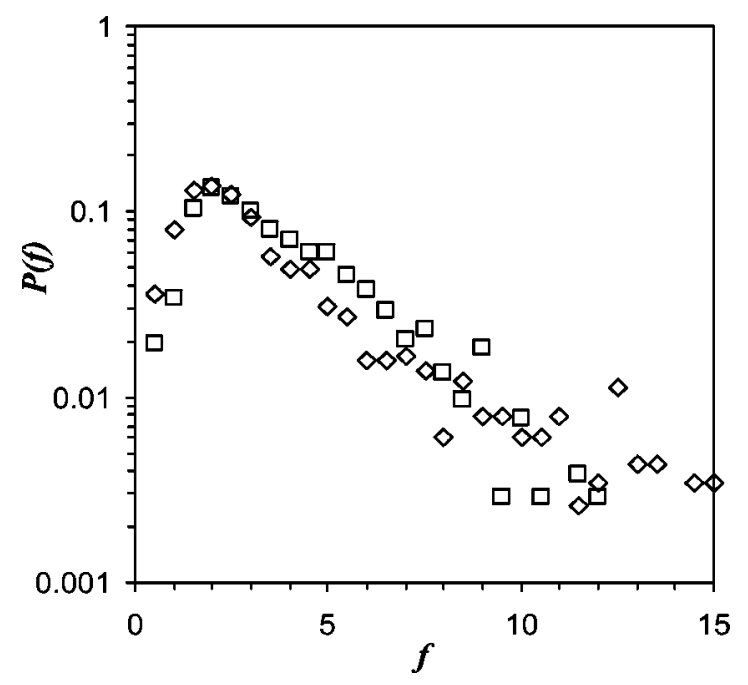

FIG. 10. Probability distribution $[P(f)]$ of the adhesion force measured from the AFM normalized to the estimated adhesion force from the bulk stresses $(f)$. Powder with 0.4 wt. $\%$ of additive (boxes). Powder with 0.05 wt. \% of additive (diamonds). 
$P$ is linear $\left(F_{t} \simeq \lambda P+F_{t 0}\right)$, we would obtain $F_{t}^{\prime} \sim \lambda P^{\prime}$ $+F_{t 0} / \eta$. Therefore, averaging the external bulk stress over all the contacts, like it is done in the derivation of Eq. (1), may lead to an underestimation of the adhesion force at a contact for a given load force. Our experimental results are compatible with this argument.

We might also argue on the effect of the sublinear dependence of the adhesion force on the load force that occurs at large loads ([7], Eq. (13)). At heavily stressed contacts (belonging to the subnetwork of force chains) the adhesion force would saturate. This would imply a reduction of the overall tensile strength as compared to the case where the external load were equally distributed over all the contacts. Another important factor would be the existence of structural defects that could also decrease the tensile strength. These defects, however, would provoke a large dispersion in the measured tensile strength contrary to experience.

\section{CONCLUSIONS}

Parallel measurements in a set of fine powders of the tensile strength $\sigma_{t}$ as a function of the consolidation stress, and of the adhesion force between individual particles $F_{t}$ as a function of the load force have been carried out. The improvement of flowability by the use of additives is reflected in a reduction of both $\sigma_{t}$ and $F_{t}$. The values measured for $F_{t}$ are in agreement with the predicted by theory assuming a plastic deformation of the contacts. We have roughly estimated from bulk stresses the interparticle contact forces $[F(\sigma)]$ neglecting anisotropy, polydispersity, and assuming the validity of a continuum description of the granular material. Despite these drastic simplifying assumptions we find a good correlation between the measured and the estimated adhesion force. The probability distribution function of the reduced adhesion force $f$ (ratio of the measured adhesion force of an individual contact to the estimated adhesion force from bulk stresses for the same load force) does not depend on the powder cohesivity, neither on the load force. The distribution has a well defined peak at $f \simeq 2$ and decays exponentially for large values of $f$. We estimate that the propagation of forces through privileged paths (chains) would raise the estimated adhesion force. Thus, these results are consistent with the existence of force chains in fine cohesive powders.

\section{ACKNOWLEDGMENTS}

This research has been supported by the Xerox Foundation, Spanish Government Agency Ministerio de Ciencia y Tecnologia (DGES) under Contract No. BMF2000-1056, and NATO Grant No. LINKAGE PST.CLG.976575.

\section{APPENDIX: THE EFFECT OF ANISOTROPY}

To obtain a closed formula for the interparticle contact force using Eq. (2) it is necessary to evaluate the fabric tensor $F_{i j}$ of the material, which is defined by Eq. (3). In integral form, Eq. (3) is written in terms of the solid volume fraction $\phi$, the coordination number $k$, and the contact angu- lar distribution $P(\Omega)$ (normalized to unity) in the form [11]:

$$
F_{i j}=\frac{3 \phi k}{\pi d_{p}^{2}} \int P(\Omega) n_{i} n_{j} d \Omega .
$$

For an oedometric test along the $z$ axis, and neglecting the wall effect [4], the stress tensor has the general form

$$
\sigma_{i j}=\sigma_{h} \delta_{i j}+\left(\sigma_{z}-\sigma_{h}\right) \delta_{i 3} \delta_{j 3} .
$$

Numerical simulations [17] have shown that the principal directions of the fabric tensor coincide with the principal directions of the strain-rate tensor, which has cylindrical symmetry around the $z$ axis in an oedometric test. Therefore the fabric tensor and the contact angular distribution must have cylindrical symmetry around the $z$ axis and reflection symmetry in the $x y$ plane. The contact angular distribution can be expanded in a series of spherical harmonics having this symmetry. For the sake of simplicity we consider only the first two terms in the series to estimate the effect of the anisotropy in the distribution of contact directions

$$
P(\Omega)=\mathcal{N}\left(Y_{00}+\zeta Y_{20}\right),
$$

where $\zeta$ is a constant giving the magnitude of the anisotropy of the contact angular distribution and $\mathcal{N}$ is a normalization factor. Inserting Eq. (A3) into Eq. (A1) yields

$$
F_{i j}=M \delta_{i j}+N \delta_{i 3} \delta_{j 3}
$$

for the fabric tensor, and

$$
A_{i j}=\frac{1}{M} \delta_{i j}+\left(\frac{1}{M+N}-\frac{1}{M}\right) \delta_{i 3} \delta_{j 3}
$$

for its inverse tensor, with $M$ and $N$ given by

$$
\begin{gathered}
M=\frac{\phi k}{\pi d_{p}^{2}}\left(1-\frac{\zeta \sqrt{5}}{2}\right), \\
N=\frac{\phi k}{\pi d_{p}^{2}} \frac{9 \zeta}{2 \sqrt{5}} .
\end{gathered}
$$

Using Eqs. (A2) and (A5) in Eq. (2) we obtain the (average) value of the components $F_{i}$ of the force acting on a contact in the direction $n_{i}$

$$
F_{i}=\frac{\sigma_{h}}{M} n_{i}+\left(\frac{\sigma_{z}}{M+N}-\frac{\sigma_{h}}{M}\right) \delta_{i 3} n_{3} .
$$

The normal force acting in the contact is given by

$$
F_{n}=F_{i} n_{i}=\frac{\sigma_{h}}{M}+\left(\frac{\sigma_{z}}{M+N}-\frac{\sigma_{h}}{M}\right) \cos ^{2} \theta
$$

where $\theta$ is the angle sustained between the contact direction and the $z$ axis. 
In the measurements of the adhesion force between two particles using the atomic force microscope we checked that the centers of the probe and substrate particles were approximately collinear in the vertical direction. This means that the vertical direction was close to the normal to the surfaces of the area of contact. Therefore, the measured values of the load and adhesion forces must be compared with the esti- mated contact forces from Eq. (A9) for which $\theta \simeq 0$. As long as $\sigma_{h}<\sigma_{z}$, Eq. (A9) can be approximated by

$$
F_{n}=F_{i} n_{i}=\frac{\pi d_{p}^{2}}{\phi k} \sigma_{z}\left(1+\frac{2}{\sqrt{5}} \zeta\right)^{-1} .
$$

In the absence of anisotropy, $\zeta=0$ and we recover Eq. (1).
[1] A. Castellanos, J.M. Valverde, A.T. Perez, A. Ramos, and P.K. Watson, Phys. Rev. Lett. 82, 1156 (1999).

[2] J.M. Valverde, A. Castellanos, A. Ramos, and P.K. Watson, Phys. Rev. E 62, 6851 (2000).

[3] O. Molerus, Powder Technol. 12, 259 (1975).

[4] J.M. Valverde, A. Ramos, A. Castellanos, and P.K. Watson, Powder Technol. 97, 237 (1998).

[5] J.M. Valverde, A. Castellanos, A. Ramos, A.T. Pérez, M.A. Morgan, and P.K. Watson, Rev. Sci. Instrum. 71, 2791 (2000).

[6] J.M. Valverde, A. Castellanos, and P.K. Watson, Powder Technol. (to be published).

[7] P.K. Watson, J.M. Valverde, and A. Castellanos, Powder Technol. 115, 44 (2001).

[8] U. Sindel, I. Zimmermann, D. Schaefer, and R. Reifenger, in Proceedings of the Third World Congress on Particle Technology (Institute of Chemical Enginnering, Brighton, UK, 1998), produced by Catalyst Electronic Publishing on $\mathrm{CD}$, paper no. 68.

[9] H.A. Mizes, J. Adhes. 51, 155 (1995).

[10] K. Walton, J. Mech. Phys. Solids 35, 213 (1987).

[11] F. Emeriault and C.S. Chang, Comput. Geotech. 20, 233 (1997).

[12] A. Misra and C.S. Chang, Int. J. Solids Struct. 30, 2547 (1983).

[13] B. Cambou, P. Dubujet, F. Emeriault, and F. Sidoroff, Eur. J. Mech. A/Solids 14, 255 (1995).

[14] H. Rumpf, Chem. Ing. Tech. 30, 144 (1958).

[15] A.S. Helle, K.E. Easterling, and M.F. Ashby, Acta Metall. 33, 2163 (1985).

[16] O. Tsoungui, D. Vallet, J.C. Charmet, and S. Roux, Phys. Rev. E 57, 4458 (1998).

[17] F. Radjai, D.E. Wolf, M. Jean, and J.J. Moreau, Phys. Rev. Lett. 80, 61 (1998).

[18] C. Liu, S.R. Nagel, D.A. Schecter, S.N. Coppersmith, S. Majumdar, O. Narayan, and T.A. Witten, Science 269, 513 (1995).

[19] B. Miller, C. O'Hern, and R.P. Behringer, Phys. Rev. Lett. 77, 3110 (1996).
[20] A. Ngadi and J. Rajchenbach, Phys. Rev. Lett. 80, 273 (1998).

[21] D.M. Mueth, H.M. Jaeger, and S.R. Nagel, Phys. Rev. E 57, 3164 (1998).

[22] G. Lovoll, K. Jorgen Maloy, and E.G. Flekkoy, Phys. Rev. E 60, 5872 (1999).

[23] R.P. Behringer, D. Howell, L. Kondic, S. Tennakoon, and C. Veje, Physica D 133, 1 (1999).

[24] F. Radjai, M. Jean, J.J. Moreau, and S. Roux, Phys. Rev. Lett. 77, 274 (1996).

[25] S.N. Coppersmith, C. Liu, S. Majumdar, O. Narayan, and T.A. Witten, Phys. Rev. E 53, 4673 (1996).

[26] S.J. Antony, Phys. Rev. E 63, 011302 (2001).

[27] D.L. Blair, N.W. Mueggenburg, A.H. Marshall, H.M. Jaeger, and S.R. Nagel, Phys. Rev. E 63, 041304 (2001).

[28] P.K. Watson, H. Mizes, A. Castellanos, and A.T. Perez, in Powders \& Grains 97, edited by R. Behringer and J.T. Jenkins (Balkema, Rotterdam, 1997), p. 109.

[29] P.C. Carman, Trans. Inst. Chem. Eng. 15, 150 (1937).

[30] J.A. Greenwood, Proc. R. Soc. London, Ser. A 453, 1277 (1997).

[31] H.C. Hamaker, Physica (Amsterdam) 4, 1058 (1937).

[32] H. Krupp, Adv. Colloid Interface Sci. 1, 111 (1967).

[33] L. Massimilla and G. Donsi, Powder Technol. 15, 253 (1976).

[34] T.G. Mason, A.J. Levine, D. Ertas, and T.C. Halsey, Phys. Rev. E 60, 5044 (2000).

[35] A.J. Forsyth and M.J. Rhodes, J. Colloid Interface Sci. 223, 133 (2000).

[36] S. Ross and I.D. Morrison, Colloidal Systems and Interfaces (Willey-Interscience, New York, 1988).

[37] D.S. Rimai, L.P. DeMejo, and R.C. Bowen, in Fundamentals of Adhesion and Interfaces, edited by D.S. Rimai, L.P. DeMejo, and K.L. Mittal (VSP, Zeist, The Netherlands, 1995), p. 1.

[38] B.N.J. Persson, Sliding Friction (Springer, Berlin, 1998).

[39] D. Maugis and H.M. Pollock, Acta Metall. 32, 1323 (1984).

[40] K.L. Johnson, K. Kendall, and A.D. Roberts, Proc. R. Soc. London, Ser. A 324, 301 (1971).

[41] D.H. Pan (private communication). 